\title{
Synthesis of nano particle of inorganic oxides by polymer matrix
}

\author{
P PRAMANIK \\ Department of Chemistry, Indian Institute of Technology, Kharagpur 721 302, India
}

\begin{abstract}
Ultrafine $(\leqslant 150 \mathrm{~nm})$ powders of spinels $\left[\mathrm{MFe}_{2} \mathrm{O}_{4}\right.$ where $\mathrm{M}=\mathrm{Ni}(\mathrm{il})$, $\mathrm{Co}(\mathrm{II})$ and $\mathrm{Zn(II)]}$ : rare-earth orthoferrites [ $\mathrm{RFeO}$, where $\mathrm{R}=\mathrm{Sm}$, Nd and $\mathrm{Gd}]$, and rare-earth garnets [ $\mathrm{R}_{3} \mathrm{Fe}_{3} \mathrm{O}_{12}$ where $\mathrm{R}=\mathrm{Sm}, \mathrm{Nd}$ and $\mathrm{Gd}$ ] with good purity and chemical homogeneity were prepared through two new versatile chemical routes. The first route involved the coprecipitation of the desired metal nitrates from their aqueous solution, in presence of a water soluble polymer polyvinyl alcohol (PVA), by triethyl ammonium carbonate solution. The other process involved complete evaporation of a mixture of optimum amounts of PVA and the desired aqueous metal nitrate solutions. with and without the addition of optimum amounts of urea when the mixture was evaporated to a pasty mass. In addition, detailed study on the reported potassium ferricyanide route was also carried out for the production of the rare-earth orthoferrite powders. The various precursor as well as the heat-treated mixed-oxide powders, prepared through each of the routes, were compared by the physical characterization studies involving thermal gravimetry and differential scanning calorimetry, infrared spectroscopy, $\mathrm{X}$-ray powder diffraction, transmission electron microscopy, and room temperature magnetic measurements.
\end{abstract}

Keywords. Spinel; rare-earth orthoferrites; rare-earth garnets; ultrafine powders; chemical route: PVA: triethylammonium carbonate: potassium ferricyanide.

\section{Introduction}

The chemical synthesis route plays a crucial role in designing the final products and is also better and less cumbersome method for the production of fine-grained mixedoxide powders. The benefit that can be obtained from the use of a controlled chemical processing route is well recognized (Uhlmann et al 1984), especially for multicomponent system. The use of chemistry in the preparation of materials can avoid three major problems - diffusion, impurities and agglomeration. The reason being that the fine chemically prepared powders allow for shorter diffusion distance and improved homogeneity, the chemical precursors used can be easily refined to increase the purity and careful control of solvent removal from the precursors will lead to the production of crushable agglomerate. However the chemical preparative routes generally involve more complex methods compared to the conventional ceramic routes and an improved level of skill is required for realizing the benefits. A variety of chemical synthesis routes exist for the preparation of fine grained mixed-oxide powders such as the precursor compound methods, the coprecipitation methods, the sol-gel methods, the solvent evaporation methods and the hydrothermal methods.

\section{Experimental}

In the present investigation, the author has developed the following versatile chemical synthesis routes for the preparation of fine-grained mixed-oxide powders. However, the author reports the synthesis of only the ferrite (spinel ferrites, iron garnets and orthoferrites) systems using these routes. The routes are: (i) Chemical coprecipitation using triethylammonium-carbonate (or, TEAC) solution, (ii) evaporation of the PVA 
added mixed-metal-nitrate solution, in the presence and in the absence of optimum amounts of urea (i.e. (PVA + urea) and (PVA, nurea) methods respectively.

In addition, the following route has also been used by the author for the preparation of fine orthoferrite powders: (iii) ferricyanide precursor route.

\subsection{TEAC coprecipitation method}

The origin of TEAC method is from an attempt to modify the existing chemical coprecipitation routes. In the present method, an organic carbonate solution has been used as the precipitant in lieu of the traditionally used inorganic salt solutions where a proper $\mathrm{pH}$ control is essential for obtaining unsegregated product. Moreover, the use of inorganic salts (such as ammonium-hydroxides, -carbonates, -oxalates etc), are unsuitable as precipitants for mixed-oxide systems containing $\mathrm{Cu}(\mathrm{II}), \mathrm{Zn}(\mathrm{II}), \mathrm{Cd}(\mathrm{II})$, and $\mathrm{Ni}(\mathrm{II})$ ions. This is because these mentioned metal ions tend to form soluble complexes with ammonia and pass into the filtrate, subsequently changing the stoichiometry of the precursors. On the other hand, use of other inorganic salt solutions such as $\mathrm{NaOH} / \mathrm{KOH}, \mathrm{Na}_{2}\left(\mathrm{C}_{2} \mathrm{O}_{4}\right) /$ $\mathrm{K}_{2}\left(\mathrm{C}_{2} \mathrm{O}_{4}\right)$ etc may result in contaminated precursor materials. Since the $\mathrm{Na}^{+} / \mathrm{K}^{+}$ions tend to get occluded into the precursor matrix, their removal required extensive washings of the precursor material which enhances the possibility of loss of certain desirable cations from the system and consequently, the stoichiometry of the final product may get affected.

The use of organic salt solutions as precipitant overcomes the problems of contaminations of the precursor materials and hence also the tedious washings. The organic component, being volatile gets easily removed from the precursor material during calcination. Their removal during the calcination of the precursor material, adds to the amount of gases evolved during the process and thus facilitates the generation of powders with finer particles. Literature reports the use of triethylammonium-oxalate (Pramanik et al 1988) as a precipitant for a variety of mixed-oxide systems. However, this coprecipitation process requires a delicate control of exact oxalate ion concentration. Even a slight excess of oxalate ions in the mixture gives rise to soluble complexes of the metal oxalates, due to the formation of stable anionic complexes. And, even the multidented network structure of the organic oxalate fails to anchor all the metal ions, resulting in a possibility of leaching of the ions of the transition metals (such as: $\mathrm{Cu}(\mathrm{II}), \mathrm{Zn}(\mathrm{II}), \mathrm{Ni}(\mathrm{II}), \mathrm{Cd}(\mathrm{II})$, etc) from the precipitate. The TEAC solution is a mixture of distilled triethylamine, ethyl alcohol, water, and $\mathrm{CO}_{2}$. Apart from having all the advantages of an organic based precipitant, the TEAC coprecipitating reagent has several other advantages. These are as follows:

(I) In the TEAC coprecipitation process, the solutions are mixed rapidly and the desired $\mathrm{pH}$ is reached more or less instantaneously. Thus, the highest level of supersaturation for each of the elements is achieved simultaneously and their segregation in the product is largely circumvented, provided the constituent cations do not have widely differing solubilities.

(II) A precise control of carbonate ion concentration is not required in the system i.e. an excess of the triethylammonium-carbonate in the mixture does not affect the final stoichiometry of the product.

(III) The triethylamine used in the preparation of TEAC solution, is less costly and commercially more viable than other higher order amines, such as tributyl- or tripropyl-amine, and this adds to the viability of the TEAC solution for large scale preparation of the mixed-oxide precursors. 
(IV) The recovery process of triethylamine from the filtrate is relatively simple and this adds to the process economics.

In this process, the desired cationic salts are taken into aqueous solution in accordance with the required stoichiometry and thoroughly mixed in the presence of polyvinyl alcohol (PVA) (about 5\% with respect to the predicted theoretical yield of the desired mixed oxide).

The precipitation is carried out by pouring the cationic mixture (heated at $70^{\circ} \mathrm{C}$ ) into the required amounts of TEAC solution (heated at $70^{\circ} \mathrm{C}$ ) with vigorous stirring. For complete precipitation, theoretically one mole of triethylammonium-carbonate is required for every gram-ion of metal present of valency two and every $2 / 3$ gram-ion metal of valency three. However, in practice the TEAC solution is always kept in excess ( 1.5 to 2 times), to compensate for any dilution of the precipitating reagent as a consequence of volatilization of $\mathrm{CO}_{2}$ gases from the system during heating. The resulting mixture is then put to heat to just before boiling with thorough stirring for $1 \mathrm{~h}$ and then the precipitate allowed to settle. After settling of the precipitate, it is separated through filtration. The mixed metal carbonate precipitate is dried in an air-oven at about $150^{\circ} \mathrm{C}$ and ground to fine powders. This resulting precursor yields the fine powders of the desired oxide-system on thermal decomposition.

The chemical reaction involved can be represented as follows:

$$
\mathrm{M}^{n+}+\left[\left(\mathrm{C}_{2} \mathrm{H}_{5}\right)_{3} \mathrm{NH}\right]_{2} \mathrm{CO}_{3} \longrightarrow \mathrm{M}_{2}\left(\mathrm{CO}_{3}\right)_{n}+\left[\left(\mathrm{C}_{2} \mathrm{H}_{5}\right)_{3} \mathrm{NH}\right]^{+},
$$

where $\mathrm{M}^{n+}$ represent the metal ion present in the system.

2.1a Role of PVA: The presence of the water soluble polymer, PVA, in the system during the precipitation process, using triethylammonium-carbonate, is expected to offer a three-dimensional polymeric network environment for the precursor nuclei. The precursor nuclei get embedded in this structure and consequently the formation of a three-dimensional network of the mixed-metal-carbonate complex is expected. This is supported by the observed colloidal nature of the precipitate.

2.1b Role of ethyl alcohol in TEAC solution: The presence of ethyl alcohol in the TEAC solution plays a dual role. It helps to keep the triethylammonium-carbonate (which is a mixture of distilled triethylamine, water, ethyl alcohol and $\mathrm{CO}_{2}$ gas) in a homogeneous solution form. Additionally it also helps the colloidal form of the mixed metal carbonate precipitate and also prevents their loss from the system during the filtering process.

Pathak and Pramanik (1992) prepared a series of ceramic mixed-oxide systems through this route which include ceramic superconductors $\left(\mathrm{YBa}_{2} \mathrm{Cu}_{3} \mathrm{O}_{x}\right.$ and $\mathrm{Bi}_{1.6} \mathrm{~Pb}_{0.4} \mathrm{Sr}_{2} \mathrm{Ca}_{2} \mathrm{Cu}_{3} \mathrm{O}_{x}$ ), spinels, orthoferrites, and garnets. However, only the studies on the spinels $\left[\mathrm{MFe}_{2} \mathrm{O}_{4}\right.$ where $\mathrm{M}=\mathrm{Ni}(\mathrm{II}), \mathrm{Co}(\mathrm{II})$, and $\left.\mathrm{Zn}(\mathrm{II})\right]$, the orthoferrites $\left[\mathrm{RFeO}_{3}\right.$ where $R=\mathrm{Nd}(\mathrm{III}), \mathrm{Sm}(\mathrm{III})$, and $\left.\mathrm{Gd}(\mathrm{III})\right]$ and the garnets $\left[\mathrm{R}_{3} \mathrm{Fe}_{5} \mathrm{O}_{12}\right.$, where $\mathrm{R}=\mathrm{Y}(\mathrm{III}), \mathrm{Sm}(\mathrm{III})$, and $\mathrm{Gd}(\mathrm{III})]$, are included in this paper.

2.2 Polyvinyl alcohol added mixed-metal solution evaporation method: (in the presence of urea (PVA+ urea) and in the absence of urea (PVA. nurea))

Polyvinyl alcohols (PVA) are widely used as binders for pelletization and moulding of various mixed-oxide powders. The PVA easily decomposes exothermally at low ignition 
temperature $\left(500^{\circ} \mathrm{C}\right)$, leaving behind very little carbon residue. The thermal gravimetry and differential scanning calorimetry (TG/DSC) curves have confirmed it. This characteristic of PVA along with its water soluble nature has motivated the initiation of this route. The route involves evaporation of a viscous PVA added mixed-metal-nitrate solution (between $130^{\circ} \mathrm{C}$ to $150^{\circ} \mathrm{C}$ ) followed by calcination of the evaporated mass.

In case of the urea added process, the urea (by weight) is introduced into the system with thorough stirring, when the PVA-added mixed metal-nitrate solution evaporates to a pasty mass. The optimum urea to cation ratio is $1: 1$. Excess of urea in the system is observed to have a detrimental effect on the grain-size due to larger heat of calcination of the product. In this process, appropriate amounts of the desired metal-nitrates are taken into aqueous solution. They are thoroughly mixed and $10 \%(\mathrm{w} / \mathrm{v})$ aqueous solution of PVA is then added to it, maintaining the desired metal ion to vinyl alcohol monomer unit of PVA mole ratio. The molecular weight of the polyvinyl alcohols (PVA) used for the present study is 1,25,000 Dalton. The PVA helps the homogeneous distribution of the metal ions in its polymeric network structure (by coordination through the secondary alcoholic groups), and inhibits their segregation/precipitation from the solution. The resulting viscous liquid mixture of PVA-metal-nitrate solution is gradually evaporated by heating between $130^{\circ} \mathrm{C}$ to $150^{\circ} \mathrm{C}$, over a hot plate or spray dryer.

The PVA, in presence of nitric acid in the solution medium, gets oxidized to the corresponding ketone, and at elevated temperatures it is ultimately converted to the carboxylic acid with simultaneous $\mathrm{C}-\mathrm{C}$ bond breaking. As evaporation proceeds, the nitrate salts also decompose with simultaneous liberation of brown fumes of $\mathrm{NO}_{2}$. On complete evaporation of the PVA-added mixed-metal-nitrate solution (in presence/ absence of urea), a voluminous, carbonaceous fluffy mass results, which is presumed to be constituted of metal carboxylates (which is expected to be formed in situ, from the reaction of the metal ions with the residue of the decomposed PVA), metal-oxides, undecomposed metal nitrates, and carbon. This composition is changeable depending on various experimental conditions such as: sample bed-temperature during the complete evaporation process, nature of metal ions present in the system, amount of PVA in the starting solution etc.

On the basis of the experimental findings, the following tentative reaction mechanism has been proposed for the decomposition of PVA and the subsequent in situ metal carboxylate formation:

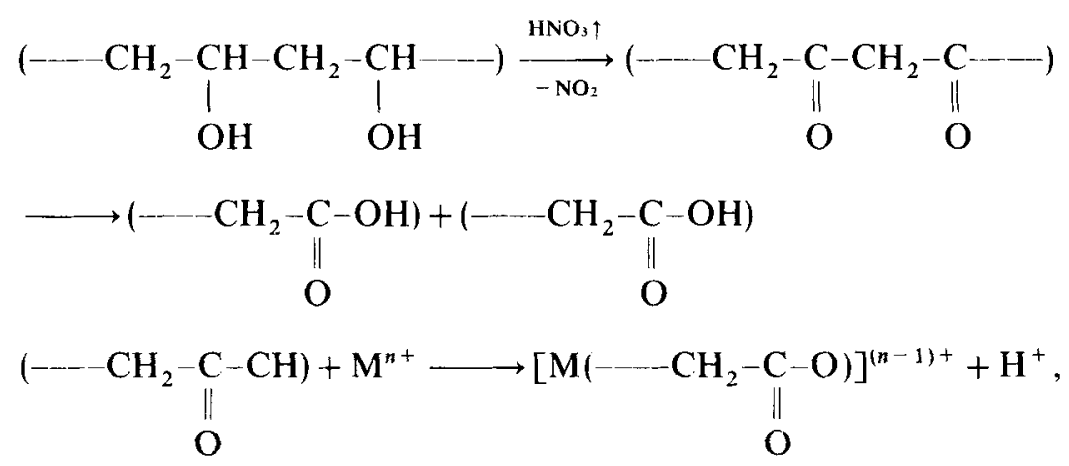

where $\mathrm{M}^{n+}$ represents the metal ion present in the system.

In presence of urea in the system, the urea, which is added when the PVA added metal-nitrate solution evaporates to a pasty mass, is expected to decompose completely 
during the complete evaporation process. The decomposition of urea can be given by the following equation:

$$
\mathrm{H}_{2} \mathrm{~N}-\mathrm{CO}-\mathrm{NH}_{2} \stackrel{\Delta}{\longrightarrow} \mathrm{NH}_{3}+\mathrm{CO}_{2}+\mathrm{HNCO}
$$

The liberation of ammonia, during the urea decomposition process, shifts the $\mathrm{pH}$ of the mixture (of the residual PVA and metal-nitrates) towards higher side $(\mathrm{pH}>7)$. This altered, slightly alkaline environment, suppresses the in situ decomposition of the metal-nitrates as well as the reaction between the residual PVA and the nitrate ions during the complete evaporation process (at temperatures less than $150^{\circ} \mathrm{C}$ ). The qualitative composition of the completely evaporated carbonaceous fluffy mass obtained through the PVA-mixed-metal-nitrate evaporation process in the presence of urea, does not differ much from that obtained in the absence of urea, except for the incorporation of some additional nitrate salts.

The voluminous carbonaceous fluffy mass obtained from the complete evaporation of the PVA-added mixed-metal-nitrate solution, in the presence/absence of urea, when ground to fine powders, produces the precursor powders. These precursor powders yield the fine powders of the desired oxide system on thermal decomposition. The high in situ temperature, attained during the exothermic decomposition of the precursor powder, facilitates the solid-phase reaction between the constituent metal ions. As a result the desired oxide-phase formation occurs at relatively low external temperatures. The exothermic decomposition of the precursor powder material is accompanied by the evolution of various gases. The evolution of gases not only helps the particles to disintegrate but also helps to dissipate the heat of combustion, thus inhibiting sintering of the fine particles.

A series of mixed-oxide systems including calcia, yttria-, magnesia-stabilized zirconia, lead zirconate titante, lanthanum modified lead zirconate titante, spinels, orthoferrites, and garnets, have been prepared through this route (Pramanik and Pathak 1994; Pramanik et al 1995).

\subsection{Ferricyanide precursor method}

This method is not new. It was first reported by Gallagher (1968), but detailed studies on particle size were not carried out. In the present investigation, an attempt has been made to make a detailed study on this route. The results obtained were from the characterization of the fine powders of orthoferrites obtained from the TEAC coprecipitation and PVA-mixed-metal-nitrate solution evaporation route.

In this process, the required amount of rare-earth-metal-nitrate aqueous solution (heated at $70^{\circ} \mathrm{C}$ ) is added to the appropriate amount of potassium ferricyanide solution (heated at $70^{\circ} \mathrm{C}$ ) with vigorous stirring. Fine crystalline precipitate of $\mathrm{R}\left[\mathrm{Fe}(\mathrm{CN})_{6}\right]$ $\mathrm{XH}_{2} \mathrm{O}$ is obtained. The precipitate is gently washed with deionized water, to remove the $\mathrm{KNO}_{3}$ solution that may be occluded in the precipitate. The precipitate is finally washed with acetone. The precursor compound is thermally decomposed during calcination to yield the fine powders of the respective orthoferrites system.

The chemical reaction involved in the formation of the metal-ferricyanide-precursor material is as follows:

$$
\mathrm{R}\left(\mathrm{NO}_{3}\right)_{3}+\mathrm{K}_{3}\left[\mathrm{Fe}(\mathrm{CN})_{6}\right] \longrightarrow \mathrm{R}\left[\mathrm{Fe}(\mathrm{CN})_{6}\right]+3 \mathrm{KNO}_{3} .
$$




\section{Results and discussion}

Characterization of the ultrafine mixed-oxide precursor materials, which were obtained through each of the mentioned chemical routes, has been done by the simultaneously recorded thermal gravimetry and differential scanning calorimetry (TG/DSC) studies. The various mixed-oxide precursors, as well as the subsequently heat treated mixed-oxide powders were further characterized by infrared (IR) spectroscopy studies between 400 and $4000 \mathrm{~cm}^{-1}$ region, by X-ray powder diffraction (XRD) studies, by transmission electron microscopy (TEM) studies and by room temperature magnetic studies using a vibrating sample magnetometer (VSM). The particle size of the various mixed-oxide powders, at various heat-treatment temperatures, were determined by $\mathrm{X}$-ray line broadening and TEM studies.

As a specimen system, the particle size of nickel-iron ferrite (NIF) produced in different reaction conditions are summarized in table 1.

The TEM particle size measurement studies of the low temperature $\left(450^{\circ} \mathrm{C}\right)$ heattreated NIF powders, prepared through the mentioned methods, indicate that the TEAC method results in relatively finer particles $(10-12 \mathrm{~nm})$ than the particles obtained through the PVA solution evaporation route. It is also evident that the addition of optimum amount of urea in the PVA solution evaporation route [i.e. 6 PVA + urea) or (2.5 PVA + urea) methods]. The study also reveals that the 6 PVA method results in relatively coarser particles compared to the particles from the 2.5 PVA method. However at high heat-treatment temperature $\left(900^{\circ} \mathrm{C}\right)$, all the powders attain similar values of the particle sizes.

\subsection{Spinels}

From the studies, it has been observed that the precursors of the spinel ferrites, obtained through the TEAC method and the PVA added solution evaporation route in presence of urea [i.e. (2.5 PVA + urea) and (6 PVA + urea)], are X-ray amorphous. On the other hand, the precursors through the (2.5 PVA, nurea) and the (6 PVA, nurea) methods, result in the respective single-phase ferrite formation in their virgin state. Whereas, the precursors through the (NPVA, nurea) method, are observed to give rise to mixed phases of the spinel ferrite, $\alpha-\mathrm{Fe}_{2} \mathrm{O}_{3}$, and the oxides of the respective bivalent metal ions in their virgin state.

Table 1. Comparative values of NIF particle sizes obtained from XRD and TEA.

\begin{tabular}{|c|c|c|c|c|c|c|c|c|c|}
\hline \multirow{2}{*}{$\begin{array}{l}\text { Preparative } \\
\text { method }\end{array}$} & \multicolumn{3}{|c|}{$\begin{array}{l}\text { Heat-treatment } \\
\text { at } 450 \mathrm{C}(2 \mathrm{~h})\end{array}$} & \multicolumn{3}{|c|}{$\begin{array}{l}\text { Heat-treatment } \\
\text { at } 700 \mathrm{C}(2 \mathrm{~h})\end{array}$} & \multicolumn{3}{|c|}{$\begin{array}{l}\text { Heat-treatment } \\
\text { at } 900^{\circ} \mathrm{C}(2 \mathrm{~h})\end{array}$} \\
\hline & $D_{s}$ & $D_{v}$ & $D_{\mathrm{TEM}}$ & $D_{\mathrm{s}}$ & $D_{v}$ & $D_{\text {TEM }}$ & $D_{\mathrm{s}}$ & $D_{v}$ & $D_{\text {TEM }}$ \\
\hline TEM & - & - & $100-120$ & 143 & 402 & $300-350$ & 245 & 962 & $1000-1200$ \\
\hline$(2.5$ PVA, NU) & 232 & 373 & $300-400$ & 232 & 403 & - & 298 & 1827 & $1000-1200$ \\
\hline$(2.5 \mathrm{PVA}+\mathrm{U})$ & -- & - & $150-170$ & 190 & 478 & $300-400$ & 309 & 1092 & $1000-1200$ \\
\hline (6 PVA, NU) & 154 & 125 & $400-500$ & 155 & 759 & $-\cdots$ & 290 & 953 & $1100-1200$ \\
\hline$(6 P V A+U)$ & 135 & 324 & $300-400$ & 155 & 560 & - & 290 & 1259 & $900-1000$ \\
\hline
\end{tabular}

Note: $D_{\mathrm{s}}, D_{\mathrm{v}}$ and $D_{\text {TEM }}$ are diameters of the particles (in $\AA$ ) obtained through Scherrer formula, variance method and TEM respectively. 
Table 2. Comparative studies of the physical properties of the NiF powders.

\begin{tabular}{|c|c|c|c|c|}
\hline $\begin{array}{l}\text { Preparation } \\
\text { route }\end{array}$ & $\begin{array}{l}\text { Metal ion: vinyl } \\
\text { alcohol monomer } \\
\text { unit (mole ratio) }\end{array}$ & $\begin{array}{c}\text { Particle } \\
\text { size from } \\
\text { TEM (avg.) }\end{array}$ & $\begin{array}{l}\text { XRD } \\
\text { phase }\end{array}$ & $\begin{array}{c}\text { Magnetic } \\
\text { studies } \\
\left(\sigma_{\mathrm{m}} \text { in emu/g }\right)\end{array}$ \\
\hline method & (n) & $10 \cdot 12 \mathrm{~nm}$ & amorphous & $\begin{array}{l}\text { Superpara- } \\
\text { magnetic } \\
(1.9 \mathrm{emu} / \mathrm{g})\end{array}$ \\
\hline $\begin{array}{l}\text { (2.5 PVA, nurea) } \\
\text { method }\end{array}$ & $1: 2 \cdot 5$ & $30 \cdot 40 \mathrm{~nm}$ & $\begin{array}{l}\text { single } \\
\text { phase }\end{array}$ & $\begin{array}{l}\text { Ferromagnetic } \\
(32.3 \mathrm{emu} / \mathrm{g})\end{array}$ \\
\hline $\begin{array}{l}(2.5 \mathrm{PVA}+\text { urea }) \\
\text { method }\end{array}$ & $1: 2 \cdot 5$ & $1517 \mathrm{~nm}$ & amorphous & $\begin{array}{l}\text { Superpara- } \\
\text { magnetic } \\
(12 \cdot 3 \mathrm{emu} / \mathrm{g})\end{array}$ \\
\hline $\begin{array}{l}\text { (6PVA, nurea) } \\
\text { method }\end{array}$ & $1: 6$ & $40-50 \mathrm{~nm}$ & $\begin{array}{l}\text { single } \\
\text { phase }\end{array}$ & $\begin{array}{l}\text { Ferromagnetic } \\
(32.0 \mathrm{emu} / \mathrm{g})\end{array}$ \\
\hline $\begin{array}{l}\text { (6PVA + urea) } \\
\text { method }\end{array}$ & $1: 6$ & $30-40 \mathrm{~nm}$ & single & $\begin{array}{l}\text { Ferromagnetic } \\
(26.7 \mathrm{emu} / \mathrm{g})\end{array}$ \\
\hline $\begin{array}{l}\text { (NPVA, nurea) } \\
\text { method }\end{array}$ & 1:0 & - & $\begin{array}{l}\text { mixed } \\
\text { phase }\end{array}$ & -- \\
\hline
\end{tabular}

The results of the XRD, TEM, and the magnetic studies, for the $\mathrm{NiFe}_{2} \mathrm{O}_{4}$ fine powders, prepared through the various routes and calcined at $450^{\circ} \mathrm{C}$ for $4 \mathrm{~h}$, are summarized in table 2.

The spinel ferrite particles are observed to be spherical through the TEM studies. The particle sizes, calculated from the XRD line broadening and TEM studies, are observed to increase with increase in the heat-treatment temperature and time, for all the powders prepared through all the routes.

(i) In the PVA solution evaporation method, the optimum vinyl alcohol monomer unit of PVA to metal ion mole ratio is observed to be $2 \cdot 5: 1$ for the production of fine, single-phase, spinel ferrite powders.

(ii) The addition of optimum amount of urea, to the optimum PVA and metal-nitrate mixture, results in finer particles compared to its absence in the system, at low heat-treatment temperatures $(<600 \mathrm{C})$.

(iii) The EDXS studies show that, within the resolving power of instrument, the point to point homogeneity is observed to be the best for the spinel ferrite powders obtained through the TEAC method.

(iv) The superparamagnetic behaviour, observed in all the magnetic spinel powders prepared through the TEAC and the (2.5 PVA + urea) methods, vanishes with increase in the heat-treatment temperatures of the powders [beyond $600^{\circ} \mathrm{C}$ for the TEAC method and beyond $500^{\circ} \mathrm{C}$ for the (2.5 PVA + urea) method].

(v) The values of the saturation magnetization of the fine spinel ferrite particles are observed to be low. The saturation magnetization values tend to approach the bulk values with increase in the particle size through increase in the heattreatment temperatures of the powders.

(vi) TEAC (triethylammonium carbonate) is observed to be a good coprecipitating agent for the preparation of contamination-free, ultrafine, single-phase, spinel ferrite powders with good homogeneity. Through the TEAC coprecipitation method, control of the particle size can be obtained by an appropriate selection of the heat-treatment temperature and time. 
(vii) The PVA solution evaporation route is technically simpler for the preparation of fine spinel ferrite particles at low external temperatures, compared to the other routes.

Through the various studies made and the experiments conducted, it can be concluded that, of the various routes considered, the TEAC and the (2.5 PVA + urea) methods are found to be the best for the production of homogeneous, single-phase, ultrafine powders of the desired spinel ferrite systems.

\subsection{Rare earth orthoferrite systems}

The precursors of RIO materials, prepared through the TEAC and the (2.5 PVA + urea) methods, are observed to be amorphous, while the precursors through the ferricyanide method show crystalline phase corresponding to the $\mathrm{RFe}(\mathrm{CN})_{6}$ system. All the precursors, when calcined at $400^{\circ} \mathrm{C}$, result in X-ray amorphous powders. These powders are observed to gradually crystallize into the respective RIO phase with increase in the heat-treatment temperatures.

(i) The powders through the (2.5 PVA + urea) method are observed to give rise to the respective crystalline $\mathrm{RIO}$ phase at a relatively low external temperatures (around $\left.500^{\circ} \mathrm{C}\right)$ compared to the TEAC $\left(800^{\circ} \mathrm{C}\right)$ or the ferricyanide method (above $600^{\circ} \mathrm{C}$ ).

(ii) At low temperature heat-treatment of the powders, the TEAC method is observed to result in finer particles $(\approx 15-20 \mathrm{~nm})$ compared to the particles prepared through the ferricyanide method $(20-30 \mathrm{~nm})$ or, the $(2.5 \mathrm{PVA}+\mathrm{urea})$ method $(30-40 \mathrm{~nm})$. This is clearly depicted in tables $3-5$.

(iii) At high temperature heat-treatment of the powders, the particle sizes of the powders produced through the ferricyanide and the (2.5 PVA + urea) methods are observed to have similar values $(80-100 \mathrm{~nm})$, while powders through the TEAC method still result in comparatively lower particle sizes $(60-70 \mathrm{~nm})$.

(iv) The crystallite/particle agglomeration is observed to be higher in the powders which are prepared through the TEAC method and heat-treated at lower temperatures $\left(\approx 600^{\circ} \mathrm{C}\right)$ than those which are prepared through the ferricyanide or the (2.5 PVA + urea) method under similar heat-treatment conditions. The observed agglomeration decreases with increase in crystallite/particle sizes through increase in the heat-treatment temperatures. The RIO powders through the (2.5 PVA + urea) and the ferricyanide methods are observed to be porous aggregates.

(v) The magnetic studies show that RIO powders possess high magnetic anisotropy and the magnetic saturation is not attained within the maximum field of $15 \mathrm{KOe}$. The magnetization values are observed to improve with increase in the heattreatment temperatures. The powders, through the TEAC method, are observed to produce better magnetization while the magnetic anisotropy appears to be higher for the powders prepared through the ferricyanide route.

(vi) The RIO powders prepared through the ferricyanide route are observed to be the best in terms of the cation homogeneity, since an exact one-to-one cation stoichiometry can be maintained in the precursor material through the $\operatorname{RFe}(\mathrm{CN})_{6}$ compound formation. All the powders are observed to improve and attain similar degree of cation homogeneity with the increase in the heat-treatment temperatures.

Thus it can be concluded that the ferricyanide method is indeed a better method for the production of RIO powder with good homogeneity and moderately good particle 
Table 3. Crystallite sizes of the calcined $(410)\left(: 2\right.$ h) $\left.\mathrm{SmFeO}_{3}(\mathrm{SIO})\right)$ powders.

\begin{tabular}{lccc}
\hline & \multicolumn{3}{c}{ Crystallite size from d li111 line $(\AA)$} \\
Heat treatment & TFAC & Ferricyanide & $(2.5 \mathrm{PVA}+$ urea $)$ \\
temperatures & methed & method & method \\
& & & \\
$600 \mathrm{C}(2 \mathrm{~h})$ & Amorphous & Amorphous & 313 \\
$800 \mathrm{C}(2 \mathrm{~h})$ & 290 & 300 & 370 \\
$900 \mathrm{C}(6 \mathrm{~h})$ & 372 & 453 & 410 \\
$1400 \mathrm{C}(2 \mathrm{~h})$ & 455 & 508 & 509 \\
\hline
\end{tabular}

Table 4. Crystallite sizes of the calcined $(400 \mathrm{C} .2 \mathrm{~h}) \mathrm{NdFeO}_{3}$ powders.

\begin{tabular}{lccc}
\hline & \multicolumn{3}{c}{ Crystallite size from $d_{1: 1,1}$ line $(\AA)$} \\
Heat treatment & TEAC & Ferricyanide & $(2.5 \mathrm{PVA}+$ urea $)$ \\
temperatures & method & method & method \\
$600 \mathrm{C}(2 \mathrm{~h})$ & Amorphous & Amorphous & 340 \\
$900 \mathrm{C}(6 \mathrm{~h})$ & $450)$ & 510 & 510 \\
$1400 \mathrm{C}(2 \mathrm{~h})$ & $420)$ & 510 & 510 \\
\hline
\end{tabular}

Amorphous: represents that the powders are $X$-raty amorphous i.e. the crystallite sizes $<200 \AA$.

Table 5. Maximun magnetization $\left(o_{n}\right)$ values for the calcined $(400 \mathrm{C}, 2 \mathrm{~h}$ ) GIO powders obtained at $15 \mathrm{KOe}$.

\begin{tabular}{lcccc}
\hline & $\sigma_{\mathrm{m}}$ (emu/g) for the GIO powders, heat-treated at various temperatures \\
Preparative & $650 \mathrm{C}(3 \mathrm{~h})$ & $730 \mathrm{C}(3 \mathrm{~h})$ & $900 \mathrm{C}(6 \mathrm{~h})$ & $1400 \mathrm{C}(2 \mathrm{~h})$ \\
route & 1.253 & 1.345 & 1.335 & 1.310 \\
& 1.271 & 1.275 & 1.257 & 1.302 \\
TEAC & 1.328 & 1.328 & 1.053 & 1.332 \\
(2.5PVA+ urea) & & & \\
\hline
\end{tabular}

Saturation magnetization $\left(\sigma_{\mathrm{m}}\right)$ values for the GIO single crystal is equal to $1.046 \mathrm{emu} / \mathrm{g}$ (Moskvin and Sinitsyn 1973 ).

size control. The TEAC method gives rise to finer particles with better particle size control. The (2.5 PVA + urea) method is suited for the preparation of the RIO powders at relatively lower external temperatures.

\subsection{Rare earth iron garnet}

The precursors of the RIG materials, prepared through the TEAC. (2.5 PVA + urea), and (2.5 PVA, nurea) methods, are observed to be X-ray amorphous. They remain amorphous even after calcination at $450 \mathrm{C}$ for $2 \mathrm{~h}$. All the precursors are observed to crystallize into the garnet phase through an intermediate nucleating phase of the respective orthoferrite and $\alpha-\mathrm{Fe}_{2} \mathrm{O}_{3}$. Increase in the heat-treatment temperatures 
facilitates the transformation

$$
3 \mathrm{RFeO}_{3}+\alpha-\mathrm{Fe}_{2} \mathrm{O}_{3} \rightarrow \mathrm{R}_{3} \mathrm{Fe}_{5} \mathrm{O}_{12} .
$$

(i) The (2.5 PVA, nurea) route realizes the formation of almost pure RIG system at faster rates and at lower external temperatures (around $900^{\circ}$ ) compared to the other two methods. However, through all the routes the RIG phase is realized as the dominant oxide phase after heat-treatment around $800^{\circ} \mathrm{C}$ to $900^{\circ} \mathrm{C}$.

(ii) With increase in the heat-treatment temperatures up to $1100^{\circ} \mathrm{C}$, the crystallite/particle sizes show an increase along with an increase in the fraction of the RIG phase in all the systems.

(iii) The average particle sizes observed from TEM at low heat-treatment temperature $\left.(60)^{\circ} \mathrm{C}\right)$ of the RIG powders are $20-25 \mathrm{~nm}$ for the TEAC method, around $30 \mathrm{~nm}$ for the (2.5 PVA + urea) method, and about $35 \mathrm{~nm}$ for the (2.5 PVA, nurea) method. High temperature $\left(900^{\circ} \mathrm{C}\right)$ heat-treatment of the RIG powders, results in higher particle sizes: $60-70 \mathrm{~nm}$ for the TEAC method, and $120-140 \mathrm{~nm}$ for the (2.5 PVA + urea) and the (2.5 PVA, nurea) methods. The IEM studies also reveal that distributions in particle sizes for the RIG powders prepared through all the three routes are narrow.

(iv) At low heat-treatment temperatures $\left(600^{\circ} \mathrm{C}\right)$, the agglomeration observed is high for the powders through the TEAC method, while the (2.5 PVA + urea) and (2.5 PVA, nurea) methods result in porous agglomerates. With heat-treatments at higher temperatures $\left(900^{\circ} \mathrm{C}\right)$ the RIG powders through the TEAC method result in the formation of porous-clusters, and those prepared through the (2.5 PVA + urea) and (2.5 PVA, nurea) methods result in the formation of ragged and angular particles with agglomeration to some extent.

(v) The room temperature magnetic measurements show that TEAC method results in RIG powders with much lower values of maximum magnetization $\left(\sigma_{m}\right.$, at $12 \mathrm{KOe}$ ) compared to the RIG powders through the PVA evaporation routes. The magnetization values for all the RIG systems are low at low heat-treatment temperature $\left(600^{\circ} \mathrm{C}\right)$. The values, in general, are observed to increase within the heat-treatment temperatures and approach the saturation magnetization of the bulk sample.

(vi) The highest values of maximum magnetization $\left(\delta_{\mathrm{m}}\right)$ at $12 \mathrm{KOe}$, obtained for the RIG powders, which are prepared through the TEAC, (2.5 PVA + urea) and the (2.5 PVA, nurea) routes, follow the following order:

$$
\left(\sigma_{\mathrm{m}}\right)_{\text {TEAC }}<\left(\sigma_{\mathrm{m}}\right)_{(2.5 \text { PVA. urea })}<\left(\sigma_{\mathrm{m}}\right)_{(2.5 \text { PVA. nurea })} .
$$

Of all the three routes, (2.5 PVA, nurea) method results in almost single phase RIG system with highest formation rate and at the lowest temperature $\left(900^{\circ} \mathrm{C}\right)$. These powders also result in the highest values of magnetization, with particle sizes ranging between $120-140 \mathrm{~nm}$. The TEAC method results in finest particles $(60-70 \mathrm{~nm})$ though the fraction of RIG phase formation is lower than the PVA evaporation routes.

\section{Acknowledgements}

The author is grateful to the Department of Science and Technology, New Delhi, for funding this investigation. He also expresses thanks to Ms Amita Pathak for her active participation in this study. 


\section{References}

Gallagher P K 1968 Mater. Res. Bull. 3225

Moskvin A S and Sinitsyn E V 1973 Soriet Phys. Solid State 142363

Pathak A and Pramanik P 1992 Mater. Mamufac. Process. 8491

Pramanik P. Biswas S. Singh C. Bhattacharya D. Dey T K, Sen D. Ghatak S K and Chopra K L 1988 Mater. Re's. Bull. 231693

Pramanik P and Pathak A 1994 Bull. Mater. Sci. 17967

Pramanik P. Saha S K and Pathak A 1995 Mater. Sci. Lett. 1435

Uhlmann D R. Zelinsky B J and Wnek G E 1984 Mattr. Res. Soc. Simp. Proc. 3259 